\section{Crystal structure of 2-(3-bromophenyl)- 1,3-dithiane}

\author{
Julio Zukerman-Schpector, ${ }^{\mathrm{a} *}$ Ignez Caracelli, ${ }^{\mathrm{b}}$ Hélio A. \\ Stefani, ${ }^{c}$ Olga Gozhina ${ }^{c}$ and Edward R. T. Tiekink ${ }^{d}$
}

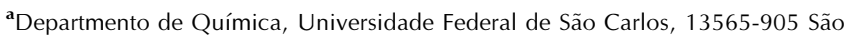
Carlos, SP, Brazil, b Departmento de Física, Universidade Federal de São Carlos, 13565-905 São Carlos, SP, Brazil, 'Departamento de Farmácia, Faculdade de Ciências Farmacêuticas, Universidade de São Paulo, 05508-900 São Paulo-SP, Brazil, and dDepartment of Chemistry, University of Malaya, 50603 Kuala Lumpur, Malaysia. *Correspondence e-mail: julio@power.ufscar.br

Received 4 February 2015; accepted 10 February 2015

Edited by P. C. Healy, Griffith University, Australia

In the title compound, $\mathrm{C}_{10} \mathrm{H}_{11} \mathrm{BrS}_{2}$, the 1,3-dithiane ring has a chair conformation with the 1,4-disposed $\mathrm{C}$ atoms being above and below the remaining four atoms. The bromobenzene ring occupies an equatorial position and forms a dihedral angle of $86.38(12)^{\circ}$ with the least-squares plane through the 1,3dithiane ring. Thus, to a first approximation the molecule has mirror symmetry with the mirror containing the bromobenzene ring and the 1,4-disposed $\mathrm{C}$ atoms of the 1,3-dithiane ring. In the crystal, molecules associate via weak methylenebromobenzene $\mathrm{C}-\mathrm{H} \cdots \pi$ and $\pi-\pi[C g \cdots C g=3.7770$ (14) $\AA$ for centrosymmetrically related bromobenzene rings] interactions, forming supramolecular layers parallel to [10 $\overline{1}$; these stack with no specific intermolecular interactions between them.

Keywords: crystal structure; 1,3-dithiane; conformation; $\mathrm{C}-\mathrm{H} \cdots \pi$ interactions; $\pi-\pi$ interactions.

CCDC reference: 1048592

\section{Related literature}

For the original synthesis and characterization of the title compound, see: Ballesteros et al. (2005). For the structure of the unsubstituted parent compound which is virtually superimposable on the title compound, see: Kalff \& Romers (1966).

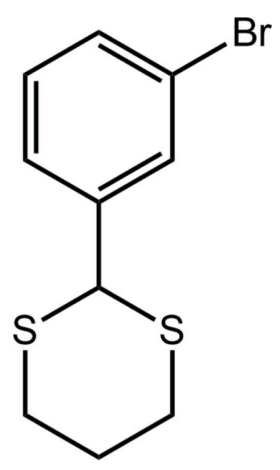

\section{Experimental}

\subsection{Crystal data}

$\mathrm{C}_{10} \mathrm{H}_{11} \mathrm{BrS}_{2}$

$M_{r}=275.22$

Monoclinic, $P 2_{b} / c$

$a=8.9821(4) \AA$

$b=11.3871(5) \AA$

$c=11.0550(5) \AA$

$\beta=99.604(3)^{\circ}$

$$
\begin{aligned}
& V=1114.86(9) \AA^{3} \\
& Z=4 \\
& \text { Mo } K \alpha \text { radiation } \\
& \mu=4.01 \mathrm{~mm}^{-1} \\
& T=296 \mathrm{~K} \\
& 0.33 \times 0.28 \times 0.16 \mathrm{~mm}
\end{aligned}
$$

\subsection{Data collection}

Bruker APEXII CCD diffractometer

Absorption correction: multi-scan (SADABS; Sheldrick, 1996) $T_{\min }=0.374, T_{\max }=0.745$

7307 measured reflections 2060 independent reflections 1820 reflections with $I>2 \sigma(I)$ $R_{\text {int }}=0.029$

\subsection{Refinement}

$R\left[F^{2}>2 \sigma\left(F^{2}\right)\right]=0.031$

$w R\left(F^{2}\right)=0.082$

$S=1.06$

2060 reflections

118 parameters

$\mathrm{H}$-atom parameters constrained

$\Delta \rho_{\max }=0.54{\mathrm{e} \AA^{-3}}^{-3}$

$\Delta \rho_{\max }=0.54 \mathrm{e} \AA^{-3}$
$\Delta \rho_{\min }=-0.65 \AA^{-3}$

Table 1

Hydrogen-bond geometry $\left(\AA,{ }^{\circ}\right)$.

$\mathrm{Cg} 1$ is the centroid of the $\mathrm{C} 5-\mathrm{C} 10$ ring.

\begin{tabular}{lccll}
\hline$D-\mathrm{H} \cdots A$ & $D-\mathrm{H}$ & $\mathrm{H} \cdots A$ & $D \cdots A$ & $D-\mathrm{H} \cdots A$ \\
\hline $\mathrm{C} 2-\mathrm{H} 2 \mathrm{~b} \cdots C g 1^{\mathrm{i}}$ & 0.97 & 2.83 & $3.668(4)$ & 146 \\
\hline Symmetry code: (i) $-x+1, y-\frac{1}{2},-z+\frac{3}{2}$.
\end{tabular}

Data collection: APEX2 (Bruker, 2009); cell refinement: SAINT (Bruker, 2009); data reduction: SAINT; program(s) used to solve structure: SIR2014 (Burla et al., 2015); program(s) used to refine structure: SHELXL2014 (Sheldrick, 2015); molecular graphics: ORTEP-3 for Windows (Farrugia, 2012) and DIAMOND (Brandenburg, 2006); software used to prepare material for publication: MarvinSketch (ChemAxon, 2010) and publCIF (Westrip, 2010).

\section{Acknowledgements}

We thank Professor Regina H. A. Santos from IQSC-USP for the X-ray data collection. The Brazilian agencies $\mathrm{CNPq}$ (305626/2013-2 to JZS, 306121/2013-2 to IC and 308320/2010-7 
to HAS), FAPESP (2012/00424-2 and 2013/21925-2) and CAPES are acknowledged for financial support.

Supporting information for this paper is available from the IUCr electronic archives (Reference: HG5429).

\section{References}

Ballesteros, L., Noguez, O., Arroyo, G., Velasco, B., Delgado, F. \& Miranda, R. (2005). J. Mex. Chem. Soc. 49, 302-306.
Brandenburg, K. (2006). DIAMOND. Crystal Impact GbR, Bonn, Germany. Bruker (2009). APEX2 and SAINT. Bruker AXS Inc., Madison, Wisconsin, USA.

Burla, M. C., Caliandro, R., Carrozzini, B., Cascarano, G. L., Cuocci, C., Giacovazzo, C., Mallamo, M., Mazzone, A. \& Polidori, G. (2015). J. Appl. Cryst. 48, 306-309.

ChemAxon (2010). Marvinsketch. http://www.chemaxon.com.

Farrugia, L. J. (2012). J. Appl. Cryst. 45, 849-854.

Kalff, H. T. \& Romers, C. (1966). Acta Cryst. 20, 490-496.

Sheldrick, G. M. (1996). SADABS. University of Göttingen, Germany.

Sheldrick, G. M. (2015). Acta Cryst. C71, 3-8.

Westrip, S. P. (2010). J. Appl. Cryst. 43, 920-925. 


\section{supporting information}

Acta Cryst. (2015). E71, o179-o180］doi:10.1107/S2056989015002832]

\section{Crystal structure of 2-(3-bromophenyl)-1,3-dithiane}

\section{Julio Zukerman-Schpector, Ignez Caracelli, Hélio A. Stefani, Olga Gozhina and Edward R. T. Tiekink}

\section{S1. Experimental}

A solution of 3-bromobenzaldehyde $(0.037 \mathrm{~mol}, 1$ equiv. $)$ in chloroform $(20 \mathrm{ml})$ was combined with an equimolar amount of propane-1,3-dithiol $(3.7 \mathrm{ml}, 0.037 \mathrm{~mol})$ at room temperature. The solution was stirred for $1 \mathrm{~h}$ at this temperature, then cooled to $-20^{\circ} \mathrm{C}$ after which $\mathrm{BF}_{3}$ etherate $(0.46 \mathrm{ml}, 0.0037 \mathrm{~mol}, 0.1$ equiv.) was added drop-wise. The reaction solution was allowed to warm to room temperature and stirred overnight. After this time, the solution was washed three times each with water, $10 \%$ aqueous $\mathrm{KOH}$, then water followed by drying over $\mathrm{MgSO}_{4}$. Evaporation of the solvent furnishes a pure product as colourless crystals in $90 \%$ yield. To obtain crystals suitable for $\mathrm{X}$-ray analysis, the product was crystallized from $\mathrm{CH}_{3} \mathrm{OH}$. The spectroscopic data matched those reported in the literature (Ballesteros et al., 2005).

\section{S2. Refinement}

Carbon-bound H-atoms were placed in calculated positions $(\mathrm{C}-\mathrm{H}=0.95-0.98 \AA)$ and were included in the refinement in the riding model approximation, with $U_{\mathrm{iso}}(\mathrm{H})=1.2 U_{\mathrm{eq}}(\mathrm{C})$. 


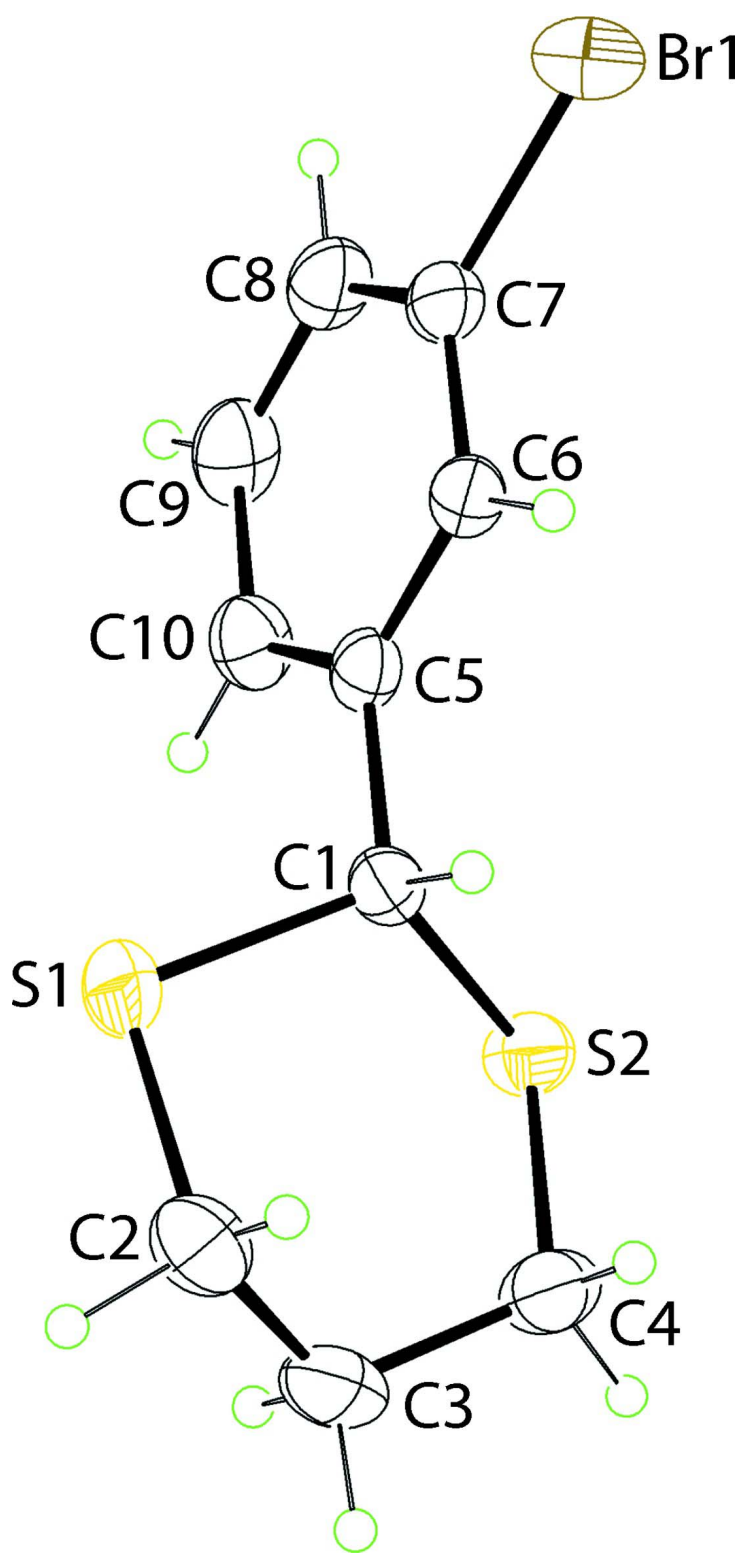

\section{Figure 1}

The molecular structure of the title compound showing the atom-labelling scheme and displacement ellipsoids at the $35 \%$ probability level. 


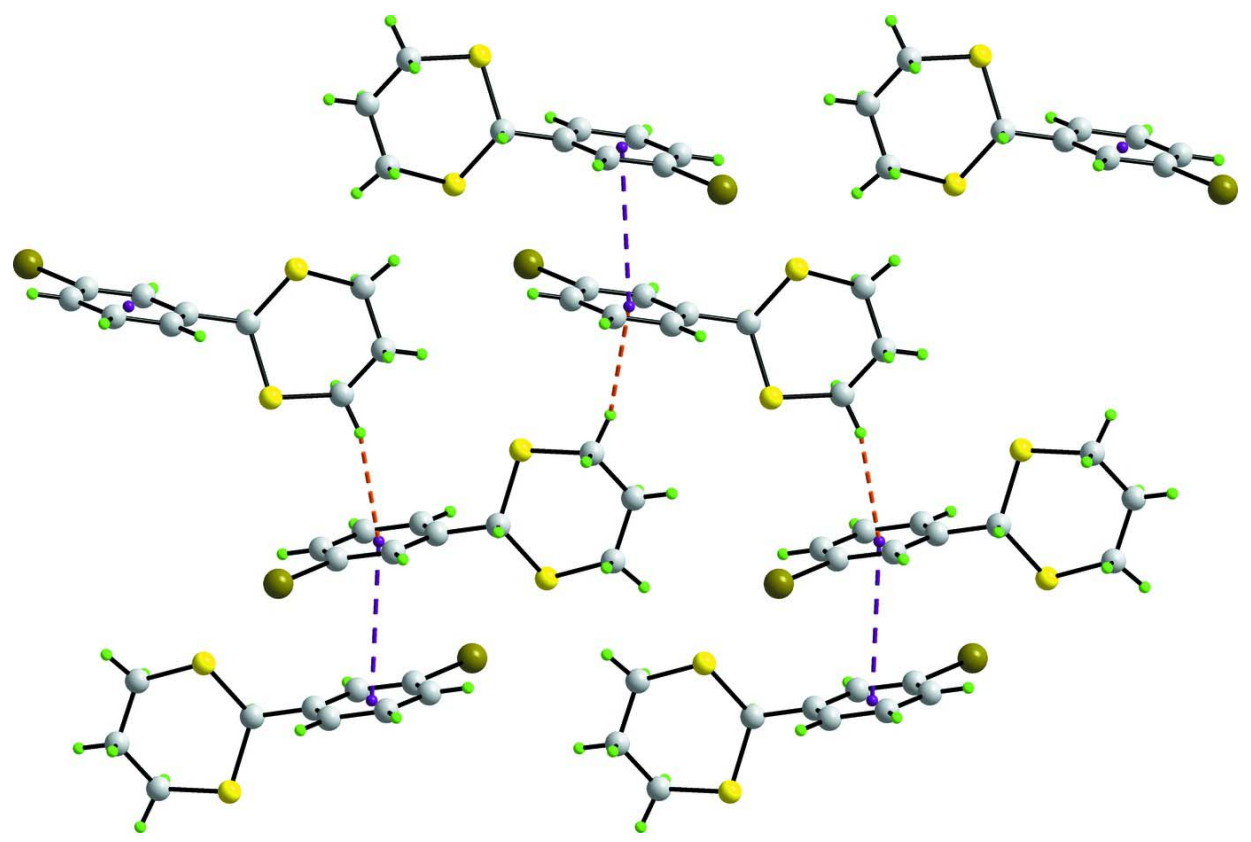

Figure 2

A view of the supramolecular layer parallel to $[10 \overline{1}]$ mediated by $\mathrm{C}-\mathrm{H} \cdots \pi$ and $\pi-\pi$ interactions shown as orange and purple dashed lines, respectively.

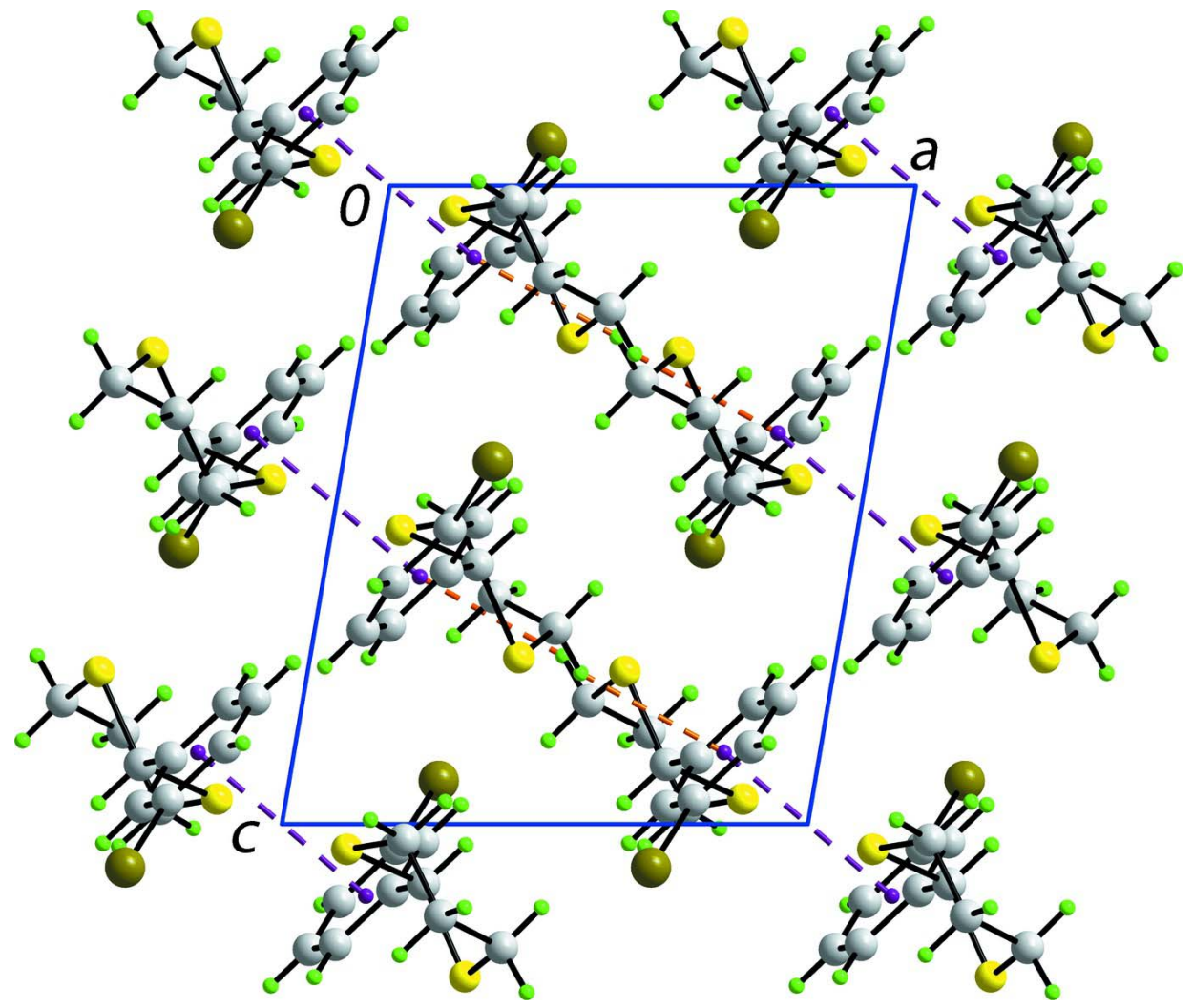

\section{Figure 3}

A view in projection down the $b$ axis of the unit-cell contents. The $\mathrm{C}-\mathrm{H} \cdots \pi$ and $\pi-\pi$ interactions shown as orange and purple dashed lines, respectively. 


\section{2-(3-Bromophenyl)-1,3-dithiane}

Crystal data

$\mathrm{C}_{10} \mathrm{H}_{11} \mathrm{BrS}_{2}$

$M_{r}=275.22$

Monoclinic, $P 2_{1} / c$

$a=8.9821(4) \AA$

$b=11.3871(5) \AA$

$c=11.0550(5) \AA$

$\beta=99.604(3)^{\circ}$

$V=1114.86(9) \AA^{3}$

$Z=4$

Data collection

Bruker APEXII CCD

diffractometer

$\varphi$ and $\omega$ scans

Absorption correction: multi-scan

(SADABS; Sheldrick, 1996)

$T_{\min }=0.374, T_{\max }=0.745$

7307 measured reflections

Refinement

Refinement on $F^{2}$

Least-squares matrix: full

$R\left[F^{2}>2 \sigma\left(F^{2}\right)\right]=0.031$

$w R\left(F^{2}\right)=0.082$

$S=1.06$

2060 reflections

118 parameters

0 restraints
$F(000)=552$

$D_{\mathrm{x}}=1.640 \mathrm{Mg} \mathrm{m}^{-3}$

Mo $K \alpha$ radiation, $\lambda=0.71073 \AA$

Cell parameters from 4286 reflections

$\theta=2.6-25.4^{\circ}$

$\mu=4.01 \mathrm{~mm}^{-1}$

$T=296 \mathrm{~K}$

Prism, colourless

$0.33 \times 0.28 \times 0.16 \mathrm{~mm}$

2060 independent reflections

1820 reflections with $I>2 \sigma(I)$

$R_{\text {int }}=0.029$

$\theta_{\text {max }}=25.4^{\circ}, \theta_{\min }=2.3^{\circ}$

$h=-9 \rightarrow 10$

$k=-13 \rightarrow 12$

$l=-13 \rightarrow 13$

Hydrogen site location: inferred from neighbouring sites

$\mathrm{H}$-atom parameters constrained

$w=1 /\left[\sigma^{2}\left(F_{\mathrm{o}}^{2}\right)+(0.0384 P)^{2}+0.6522 P\right]$

where $P=\left(F_{\mathrm{o}}{ }^{2}+2 F_{\mathrm{c}}{ }^{2}\right) / 3$

$(\Delta / \sigma)_{\max }=0.002$

$\Delta \rho_{\max }=0.54 \mathrm{e}^{-3}$

$\Delta \rho_{\min }=-0.65$ e $\AA^{-3}$

Special details

Geometry. All e.s.d.'s (except the e.s.d. in the dihedral angle between two 1.s. planes) are estimated using the full covariance matrix. The cell e.s.d.'s are taken into account individually in the estimation of e.s.d.'s in distances, angles and torsion angles; correlations between e.s.d.'s in cell parameters are only used when they are defined by crystal symmetry. An approximate (isotropic) treatment of cell e.s.d.'s is used for estimating e.s.d.'s involving 1.s. planes.

Fractional atomic coordinates and isotropic or equivalent isotropic displacement parameters $\left(\hat{A}^{2}\right)$

\begin{tabular}{lllll}
\hline & $x$ & $y$ & $z$ & $U_{\text {iso }} * / U_{\text {eq }}$ \\
\hline Br1 & $0.71577(4)$ & $1.19362(3)$ & $1.06788(3)$ & $0.06773(15)$ \\
S1 & $0.60308(9)$ & $0.70880(7)$ & $0.76097(6)$ & $0.0565(2)$ \\
S2 & $0.86833(7)$ & $0.65738(6)$ & $0.96061(6)$ & $0.04880(19)$ \\
C1 & $0.7115(3)$ & $0.7543(2)$ & $0.9063(2)$ & $0.0373(5)$ \\
H1 & 0.6439 & 0.7542 & 0.9674 & $0.045^{*}$ \\
C2 & $0.5426(4)$ & $0.5670(3)$ & $0.8068(3)$ & $0.0657(8)$ \\
H2A & 0.4799 & 0.5784 & 0.8692 & $0.079^{*}$ \\
H2B & 0.4805 & 0.5306 & 0.7366 & $0.079 *$ \\
C3 & $0.6689(4)$ & $0.4839(3)$ & $0.8560(3)$ & $0.0649(8)$ \\
H3A & 0.6262 & 0.4076 & 0.8690 & $0.078^{*}$ \\
H3B & 0.7342 & 0.4746 & 0.7950 & $0.078^{*}$
\end{tabular}




$\begin{array}{lllll}\text { C4 } & 0.7631(3) & 0.5247(3) & 0.9752(3) & 0.0587(7) \\ \text { H4A } & 0.8331 & 0.4628 & 1.0065 & 0.070^{*} \\ \text { H4B } & 0.6969 & 0.5378 & 1.0349 & 0.070^{*} \\ \text { C5 } & 0.7677(2) & 0.8778(2) & 0.8960(2) & 0.0377(5) \\ \text { C6 } & 0.7265(3) & 0.9638(2) & 0.9723(2) & 0.0382(5) \\ \text { H6 } & 0.6661 & 0.9450 & 1.0301 & 0.046^{*} \\ \text { C7 } & 0.7754(3) & 1.0778(2) & 0.9623(2) & 0.0432(6) \\ \text { C8 } & 0.8665(3) & 1.1081(3) & 0.8788(2) & 0.0526(7) \\ \text { H8 } & 0.8994 & 1.1851 & 0.8733 & 0.063^{*} \\ \text { C9 } & 0.9078(3) & 1.0224(3) & 0.8039(2) & 0.0575(7) \\ \text { H9 } & 0.9692 & 1.0417 & 0.7469 & 0.069^{*} \\ \text { C10 } & 0.8598(3) & 0.9074(3) & 0.8115(2) & 0.0496(6) \\ \text { H10 } & 0.8892 & 0.8503 & 0.7602 & 0.060^{*}\end{array}$

Atomic displacement parameters $\left(\AA^{2}\right)$

\begin{tabular}{lllllll}
\hline & $U^{11}$ & $U^{22}$ & $U^{33}$ & $U^{12}$ & $U^{13}$ & $U^{23}$ \\
\hline Br1 & $0.0883(3)$ & $0.0415(2)$ & $0.0730(2)$ & $0.00622(14)$ & $0.01259(18)$ & $-0.00728(13)$ \\
S1 & $0.0631(5)$ & $0.0554(4)$ & $0.0434(4)$ & $-0.0065(3)$ & $-0.0132(3)$ & $0.0005(3)$ \\
S2 & $0.0406(3)$ & $0.0434(4)$ & $0.0579(4)$ & $0.0002(3)$ & $-0.0047(3)$ & $0.0017(3)$ \\
C1 & $0.0393(12)$ & $0.0381(13)$ & $0.0343(12)$ & $0.0000(10)$ & $0.0053(9)$ & $-0.0026(10)$ \\
C2 & $0.0624(18)$ & $0.0590(19)$ & $0.0669(18)$ & $-0.0188(15)$ & $-0.0145(15)$ & $-0.0067(15)$ \\
C3 & $0.075(2)$ & $0.0415(16)$ & $0.073(2)$ & $-0.0107(14)$ & $-0.0016(16)$ & $-0.0100(14)$ \\
C4 & $0.0611(18)$ & $0.0409(15)$ & $0.0682(18)$ & $-0.0039(13)$ & $-0.0062(14)$ & $0.0077(13)$ \\
C5 & $0.0353(12)$ & $0.0419(13)$ & $0.0339(11)$ & $-0.0019(10)$ & $-0.0002(9)$ & $0.0037(10)$ \\
C6 & $0.0390(12)$ & $0.0402(13)$ & $0.0348(11)$ & $-0.0006(10)$ & $0.0043(9)$ & $0.0028(10)$ \\
C7 & $0.0449(14)$ & $0.0395(13)$ & $0.0412(13)$ & $-0.0015(10)$ & $-0.0041(10)$ & $0.0014(10)$ \\
C8 & $0.0559(16)$ & $0.0479(16)$ & $0.0507(15)$ & $-0.0157(13)$ & $-0.0007(12)$ & $0.0100(12)$ \\
C9 & $0.0554(17)$ & $0.072(2)$ & $0.0476(15)$ & $-0.0166(14)$ & $0.0153(13)$ & $0.0098(14)$ \\
C10 & $0.0502(15)$ & $0.0592(17)$ & $0.0414(13)$ & $-0.0046(13)$ & $0.0130(11)$ & $-0.0015(12)$ \\
& & & & & & \\
\hline
\end{tabular}

Geometric parameters $\left(\AA,{ }^{o}\right)$

\begin{tabular}{llll}
\hline $\mathrm{Br} 1-\mathrm{C} 7$ & $1.897(3)$ & $\mathrm{C} 4-\mathrm{H} 4 \mathrm{~A}$ & 0.9700 \\
$\mathrm{~S} 1-\mathrm{C} 2$ & $1.803(3)$ & $\mathrm{C} 4-\mathrm{H} 4 \mathrm{~B}$ & 0.9700 \\
$\mathrm{~S} 1-\mathrm{C} 1$ & $1.810(2)$ & $\mathrm{C} 5-\mathrm{C} 6$ & $1.383(3)$ \\
$\mathrm{S} 2-\mathrm{C} 4$ & $1.803(3)$ & $\mathrm{C} 5-\mathrm{C} 10$ & $1.389(4)$ \\
$\mathrm{S} 2-\mathrm{C} 1$ & $1.811(2)$ & $\mathrm{C} 6-\mathrm{C} 7$ & $1.381(3)$ \\
$\mathrm{C} 1-\mathrm{C} 5$ & $1.505(3)$ & $\mathrm{C} 6-\mathrm{H} 6$ & 0.9300 \\
$\mathrm{C} 1-\mathrm{H} 1$ & 0.9800 & $\mathrm{C} 7-\mathrm{C} 8$ & $1.375(4)$ \\
$\mathrm{C} 2-\mathrm{C} 3$ & $1.507(4)$ & $\mathrm{C} 8-\mathrm{C} 9$ & $1.371(4)$ \\
$\mathrm{C} 2-\mathrm{H} 2 \mathrm{~A}$ & 0.9700 & $\mathrm{C} 8-\mathrm{H} 8$ & 0.9300 \\
$\mathrm{C} 2-\mathrm{H} 2 \mathrm{~B}$ & 0.9700 & $\mathrm{C}-\mathrm{C} 10$ & $1.385(4)$ \\
$\mathrm{C} 3-\mathrm{C} 4$ & $1.515(4)$ & $\mathrm{C} 9-\mathrm{H} 9$ & 0.9300 \\
$\mathrm{C} 3-\mathrm{H} 3 \mathrm{~A}$ & 0.9700 & $\mathrm{C} 10-\mathrm{H} 10$ & 0.9300 \\
$\mathrm{C} 3-\mathrm{H} 3 \mathrm{~B}$ & 0.9700 & & 108.8 \\
$\mathrm{C} 2-\mathrm{S} 1-\mathrm{C} 1$ & & &
\end{tabular}




\begin{tabular}{|c|c|c|c|}
\hline $\mathrm{C} 4-\mathrm{S} 2-\mathrm{C} 1$ & $98.59(13)$ & $\mathrm{C} 3-\mathrm{C} 4-\mathrm{H} 4 \mathrm{~B}$ & 108.8 \\
\hline $\mathrm{C} 5-\mathrm{C} 1-\mathrm{S} 1$ & $109.74(15)$ & $\mathrm{S} 2-\mathrm{C} 4-\mathrm{H} 4 \mathrm{~B}$ & 108.8 \\
\hline $\mathrm{C} 5-\mathrm{C} 1-\mathrm{S} 2$ & $110.01(16)$ & $\mathrm{H} 4 \mathrm{~A}-\mathrm{C} 4-\mathrm{H} 4 \mathrm{~B}$ & 107.7 \\
\hline $\mathrm{S} 1-\mathrm{C} 1-\mathrm{S} 2$ & $113.16(13)$ & $\mathrm{C} 6-\mathrm{C} 5-\mathrm{C} 10$ & $119.2(2)$ \\
\hline $\mathrm{C} 5-\mathrm{C} 1-\mathrm{H} 1$ & 107.9 & $\mathrm{C} 6-\mathrm{C} 5-\mathrm{C} 1$ & $119.3(2)$ \\
\hline $\mathrm{S} 1-\mathrm{C} 1-\mathrm{H} 1$ & 107.9 & $\mathrm{C} 10-\mathrm{C} 5-\mathrm{C} 1$ & $121.5(2)$ \\
\hline $\mathrm{S} 2-\mathrm{C} 1-\mathrm{H} 1$ & 107.9 & $\mathrm{C} 7-\mathrm{C} 6-\mathrm{C} 5$ & $119.7(2)$ \\
\hline $\mathrm{C} 3-\mathrm{C} 2-\mathrm{S} 1$ & $114.8(2)$ & $\mathrm{C} 7-\mathrm{C} 6-\mathrm{H} 6$ & 120.1 \\
\hline $\mathrm{C} 3-\mathrm{C} 2-\mathrm{H} 2 \mathrm{~A}$ & 108.6 & $\mathrm{C} 5-\mathrm{C} 6-\mathrm{H} 6$ & 120.1 \\
\hline $\mathrm{S} 1-\mathrm{C} 2-\mathrm{H} 2 \mathrm{~A}$ & 108.6 & $\mathrm{C} 8-\mathrm{C} 7-\mathrm{C} 6$ & $121.5(3)$ \\
\hline $\mathrm{C} 3-\mathrm{C} 2-\mathrm{H} 2 \mathrm{~B}$ & 108.6 & $\mathrm{C} 8-\mathrm{C} 7-\mathrm{Br} 1$ & $120.0(2)$ \\
\hline $\mathrm{S} 1-\mathrm{C} 2-\mathrm{H} 2 \mathrm{~B}$ & 108.6 & $\mathrm{C} 6-\mathrm{C} 7-\mathrm{Br} 1$ & $118.53(19)$ \\
\hline $\mathrm{H} 2 \mathrm{~A}-\mathrm{C} 2-\mathrm{H} 2 \mathrm{~B}$ & 107.5 & $\mathrm{C} 9-\mathrm{C} 8-\mathrm{C} 7$ & $118.6(3)$ \\
\hline $\mathrm{C} 4-\mathrm{C} 3-\mathrm{C} 2$ & $113.5(3)$ & $\mathrm{C} 9-\mathrm{C} 8-\mathrm{H} 8$ & 120.7 \\
\hline $\mathrm{C} 4-\mathrm{C} 3-\mathrm{H} 3 \mathrm{~A}$ & 108.9 & $\mathrm{C} 7-\mathrm{C} 8-\mathrm{H} 8$ & 120.7 \\
\hline $\mathrm{C} 2-\mathrm{C} 3-\mathrm{H} 3 \mathrm{~A}$ & 108.9 & $\mathrm{C} 8-\mathrm{C} 9-\mathrm{C} 10$ & $121.2(3)$ \\
\hline $\mathrm{C} 4-\mathrm{C} 3-\mathrm{H} 3 \mathrm{~B}$ & 108.9 & $\mathrm{C} 8-\mathrm{C} 9-\mathrm{H} 9$ & 119.4 \\
\hline $\mathrm{C} 2-\mathrm{C} 3-\mathrm{H} 3 \mathrm{~B}$ & 108.9 & $\mathrm{C} 10-\mathrm{C} 9-\mathrm{H} 9$ & 119.4 \\
\hline $\mathrm{H} 3 \mathrm{~A}-\mathrm{C} 3-\mathrm{H} 3 \mathrm{~B}$ & 107.7 & $\mathrm{C} 9-\mathrm{C} 10-\mathrm{C} 5$ & $119.8(3)$ \\
\hline $\mathrm{C} 3-\mathrm{C} 4-\mathrm{S} 2$ & $113.8(2)$ & $\mathrm{C} 9-\mathrm{C} 10-\mathrm{H} 10$ & 120.1 \\
\hline $\mathrm{C} 3-\mathrm{C} 4-\mathrm{H} 4 \mathrm{~A}$ & 108.8 & $\mathrm{C} 5-\mathrm{C} 10-\mathrm{H} 10$ & 120.1 \\
\hline $\mathrm{C} 2-\mathrm{S} 1-\mathrm{C} 1-\mathrm{C} 5$ & $-175.91(19)$ & $\mathrm{S} 2-\mathrm{C} 1-\mathrm{C} 5-\mathrm{C} 10$ & $66.0(3)$ \\
\hline $\mathrm{C} 2-\mathrm{S} 1-\mathrm{C} 1-\mathrm{S} 2$ & $60.79(17)$ & $\mathrm{C} 10-\mathrm{C} 5-\mathrm{C} 6-\mathrm{C} 7$ & $0.9(3)$ \\
\hline $\mathrm{C} 4-\mathrm{S} 2-\mathrm{C} 1-\mathrm{C} 5$ & $175.20(17)$ & $\mathrm{C} 1-\mathrm{C} 5-\mathrm{C} 6-\mathrm{C} 7$ & $-179.0(2)$ \\
\hline $\mathrm{C} 4-\mathrm{S} 2-\mathrm{C} 1-\mathrm{S} 1$ & $-61.65(17)$ & $\mathrm{C} 5-\mathrm{C} 6-\mathrm{C} 7-\mathrm{C} 8$ & $-0.9(4)$ \\
\hline $\mathrm{C} 1-\mathrm{S} 1-\mathrm{C} 2-\mathrm{C} 3$ & $-58.8(3)$ & $\mathrm{C} 5-\mathrm{C} 6-\mathrm{C} 7-\mathrm{Br} 1$ & $179.66(17)$ \\
\hline $\mathrm{S} 1-\mathrm{C} 2-\mathrm{C} 3-\mathrm{C} 4$ & $65.2(4)$ & $\mathrm{C} 6-\mathrm{C} 7-\mathrm{C} 8-\mathrm{C} 9$ & $0.5(4)$ \\
\hline $\mathrm{C} 2-\mathrm{C} 3-\mathrm{C} 4-\mathrm{S} 2$ & $-65.7(4)$ & $\mathrm{Br} 1-\mathrm{C} 7-\mathrm{C} 8-\mathrm{C} 9$ & $179.9(2)$ \\
\hline $\mathrm{C} 1-\mathrm{S} 2-\mathrm{C} 4-\mathrm{C} 3$ & $60.0(3)$ & $\mathrm{C} 7-\mathrm{C} 8-\mathrm{C} 9-\mathrm{C} 10$ & $-0.2(4)$ \\
\hline $\mathrm{S} 1-\mathrm{C} 1-\mathrm{C} 5-\mathrm{C} 6$ & $120.8(2)$ & $\mathrm{C} 8-\mathrm{C} 9-\mathrm{C} 10-\mathrm{C} 5$ & $0.3(4)$ \\
\hline $\mathrm{S} 2-\mathrm{C} 1-\mathrm{C} 5-\mathrm{C} 6$ & $-114.1(2)$ & $\mathrm{C} 6-\mathrm{C} 5-\mathrm{C} 10-\mathrm{C} 9$ & $-0.7(4)$ \\
\hline $\mathrm{S} 1-\mathrm{C} 1-\mathrm{C} 5-\mathrm{C} 10$ & $-59.1(3)$ & $\mathrm{C} 1-\mathrm{C} 5-\mathrm{C} 10-\mathrm{C} 9$ & $179.3(2)$ \\
\hline
\end{tabular}

Hydrogen-bond geometry $\left(\AA,{ }^{\circ}\right)$

$\mathrm{Cg} 1$ is the centroid of the $\mathrm{C} 5-\mathrm{C} 10$ ring.

\begin{tabular}{lllll}
\hline$D-\mathrm{H} \cdots A$ & $D-\mathrm{H}$ & $\mathrm{H} \cdots A$ & $D \cdots A$ & $D-\mathrm{H} \cdots A$ \\
\hline $\mathrm{C} 2-\mathrm{H} 2 \mathrm{~b} \cdots C g 1^{\mathrm{i}}$ & 0.97 & 2.83 & $3.668(4)$ & 146 \\
\hline
\end{tabular}

Symmetry code: (i) $-x+1, y-1 / 2,-z+3 / 2$. 